\title{
Swarm intelligence and evolutionary computation approaches for 2D face recognition: a systematic review
}

\author{
Guilherme Felippe Plichoski ${ }^{1}$, Chidambaram Chidambaram ${ }^{2}$ and Rafael \\ Stubs Parpinelli ${ }^{1}$
}

${ }^{1}$ Graduate Program in Applied Computing - State University of Santa Catarina, Joinville - SC - Brazil and

${ }^{2}$ Department of Production Engineering - State University of Santa Catarina, Joinville - SC - Brazil

guilherme.plichoski@edu.udesc.br; chidambaram@udesc.br; rafael.parpinelli@udesc.br

Received: 13/03/2018. Revised: 21/06/2018. Accepted: 23/06/2018.

\begin{abstract}
A wide range of approaches for 2D face recognition (FR) systems can be found in the literature due to its high applicability and issues that need more investigation yet which include occlusion, variations in scale, facial expression, and illumination. Over the last years, a growing number of improved 2D FR systems using Swarm Intelligence and Evolutionary Computing algorithms have emerged. The present work brings an up-to-date Systematic Literature Review (SLR) concerning the use of Swarm Intelligence and Evolutionary Computation applied in 2D FR systems. Also, this review analyses and points out the key techniques and algorithms used and suggests some directions for future research.
\end{abstract}

Key words: Bio-inspired algorithms; Evolutionary algorithms; Face Recognition; Natural Computing; Optimization; Swarm algorithms

\section{Resumo}

Uma ampla gama de abordagens para sistemas de reconhecimento facial (FR) 2D pode ser encontrada na literatura devido a sua alta aplicabilidade e também por questões que necessitam de mais investigação, incluindo oclusão, variações de escala, expressão facial e iluminação. Nos últimos anos, um número crescente de sistemas de FR 2D usando algoritmos de Inteligência de Enxame e Computação Evolucionária surgiram. $\mathrm{O}$ presente trabalho traz uma Revisão Sistemática de Literatura (SLR) atualizada sobre o uso da Inteligência de Enxame e Computação Evolucionária aplicada em sistemas de FR 2D. Além disso, esta revisão analisa e aponta as principais técnicas e algoritmos utilizados e sugere alguns direcionamentos para pesquisas futuras.

Palavras-Chave: Algoritmos Bio-inspirados; Algoritmos Evolutivos; Algoritmos de Enxame; Computação Natural; Otimização; Reconhecimento Facial

\section{Introduction}

Face recognition (FR) systems are widely used in different parts of the society which includes, for example, residences, public places, industries, commercial shops and offices. In addition to this, in recent decades, some internet applications have been developed using the 2D FR systems. Generally biometric-based human recognition systems are becoming popular making possible to breach traditional security systems (Islam et al.; 2012). Hence, it is possible to find security systems based on biometric identification such as gait, fingerprint, signature, voice, iris, face recognition (FR), and others (Nebti and Boukerram; 2017). Among these, FR systems stands out due to the necessity of an identification system for real-world situations with a high flow of people. As an example, it is 
impractical to stop everyone at the entrance of an airport to record their fingerprint, but to place a camera recording the flow is enough for face capture. We have reached a point where FR systems perform well under controlled settings (Ochoa-Villegas et al.; 2015), however real-time situations require systems that can deal with uncontrolled settings like possible occlusion, adornments changing, different scales, facial expressions and illumination variation. Although we have many techniques that perform well under some uncontrolled conditions, it is worth pointing out that, up to this moment, no generic technique have been proposed to guarantee total immunity to this problems (Ochoa-Villegas et al.; 2015), leaving a gap for the research community. Among different kind of approaches proposed during the last decades, some studies can be found in literature which are focused on approaches that employ optimization techniques inspired by nature, i.e. bio-inspired optimization techniques (Bowyer et al.; 2006; Abate et al.; 2007; Islam et al.; 2012; Scheenstra et al.; 2005; Kong et al.; 2005; Zhao et al.; 2003; Alsalibi et al.; 2015).

It is possible to classify the bio-inspired algorithms into different groups based on their source of inspiration (Fister et al.; 2013). Algorithms inspired by physiochemical systems were designed to mimic the behavior and characteristics of certain laws of physics or chemistry, including gravity, electric charges, and pluvial systems. In this group we can cite Big-Bang Big-Crunch Optimization, Black Hole Optimization, Simulated Annealing, among others (Fister et al.; 2013). Algorithms based on biological systems have its source of inspiration originated from Biology. In this category we have Artificial Neural Networks (McCulloch and Pitts; 1943), Artificial Immune Systems (Dasgupta et al.; 2011), Evolutionary Computation (De Jong; 2006) and Swarm Intelligence (Parpinelli and Lopes; 2011), being the last two the focus of this work due to its applicability in FR systems. From now on, the term bio-inspired algorithms is referred in this work as any Swarm Intelligence (SI) and Evolutionary Computation (EC) approaches. Through this, we may realize that an increased number of bio-inspired FR systems had emerged due their intelligent problem-solving ability, scalability, flexibility, and adaptive nature.

SI and EC are the two major branches representing the biologically inspired algorithms for optimization. SI-based approaches are inspired by the social and collective behavior of insects, such as ants, termites, bees, flock of birds and fish school. In this branch, we can cite the Particle Swarm Optimization (PSO) algorithm (Eberhart and Shi; 2011), one of the wellknown algorithms among researchers, and it is inspired by the coordinated movement of fish schools and bird flocks. Among many versions of PSO, its binary version has been widely used to find the most discriminative set of features in facial images improving FR systems (Vora et al.; 2014; Varun et al.; 2015; Varadarajan et al.; 2015). Another popular SIbased algorithm is the Ant Colony Optimization (ACO) (Dorigo and Stutzle; 2003), which is inspired by the collective behavior of ants in finding the shortest path between the nest and the food source through a substance called pheromone. This algorithm has been used for feature selection and recognition purposes in 2D FR systems (Kanan et al.; 2007; Venkatesan and Madane; 2010; Kaur et al.; 2013a). Other SI algorithms that have been used in FR systems are inspired by bacterial foraging (BFO) (Passino; 2002), bees foraging (BA, ABC) (Pham et al.; 2011; Karaboga; 2005), brood parasitic behavior of some cuckoo species (CSA) (Yang and Deb; 2009), and gravity law and mass interactions (GSA) (Rashedi et al.; 2009). Many other SI algorithms can be found in (Parpinelli and Lopes; 2011). EC-based algorithms are inspired by the evolutionary theory proposed by Darwin. In this branch we may cite the Genetic Algorithm (GA) (Holland; 1973), in which natural selection and genetic operators play the main role, and has been used for feature selection and classification in FR systems (Fan and Verma; 2004; Zheng et al.; 2005; Liu and Wang; 2006). Another algorithm based in Darwinian theory is the Differential Evolution (DE) algorithm (Storn and Price; 1997) which has been also used as an optimization technique in FR systems (Mallipeddi and Lee; 2012; Yoo et al.; 2013).

According to Detroz et al. (2015), the traditional bibliographies are oriented by the researchers experience, which might lead to biased results. Unlike, a systematic review aims to present a fair evaluation of a research topic, identifying and evaluating in a reliable and impartial manner all relevant researches using a trustworthy, rigorous, and auditable methodology (Keele; 2007). Also, it allows to summarize the benefits and limitations of a specific method (Kitchenham; 2004). Hence, this work presents a Systematic Literature Review (SLR) of $2 \mathrm{D}$ face recognition systems using biologically inspired approaches (considering both SI and EC algorithms), providing a well-defined methodology to identify, analyze and interpret all available evidences related to the topic (Keele; 2007). After filtering the results through objective, inclusion and exclusion criteria, seventy three relevant works are gathered for SLR purpose. Some of the bio-inspired algorithms used in 2D FR systems for different purposes are template matching (Chidambaram et al.; 2012), classification (Nebti and Boukerram; 2017), parameters optimization (Oh et al.; 2016; Shen et al.; 2015; Fernández-Martínez and Cernea; 2015; Loderer and Pavlovičová; 2014), and feature selection (Khadhraoui et al.; 2016; Rao and Rao; 2016; Farag et al.; 2016).

It is important to highlight that in Alsalibi et al. (2015) a relevant review with the same scope of ours is presented. However, in the Alsalibi's work, the review period encompass papers published until 2013, and a SLR methodology is not employed. With the emergence of new bio-inspired algorithms and its approaches, along with the application of a rigorous review methodology, the present work brings the following contributions:

i. An up-to-date review of bio-inspired approaches for 2D FR Systems using the SLR methodology.

ii. The description of how each algorithm is employed in FR systems.

iii. A brief description of each work that is analyzed.

iv. A summary of employed approaches and algorithms. 
v. Exposure of how bio-inspired approaches represent a possible solution and fitness function. vi. A rank of databases with its main features.

This review is organized as follows: Section 2 describes the SLR methodology; a brief description of bio-inspired algorithms and their applications to FR systems are reported in Section 4; Section 5 presents a summary and the discussion on the review; and the conclusions and future trends are presented in Section 6.

\section{Research Method}

Petersen et al. (2008) presents the SLR methodology. The first step is to define the research questions (RQ) in order to identify and evaluate all available relevant works, which are presented as follows:

- $\mathrm{RQ}_{1}$ : What EC and SI algorithms are being applied to $2 \mathrm{D}$ FR systems?

- $\mathrm{RQ}_{2}$ : How EC and SI algorithms are being applied in $2 \mathrm{D}$ FR systems?

With the scope defined, the SLR is effectively done through the following steps: planning and conducting.

\subsection{Planning the Review}

After defining the RQs, the search for relevant works must be done. This work performs an automated search, in which it is defined a boolean search string with keywords used as input in Academic Search Engines (ASE). The following words were employed: faces, face, facial, recognition, detection, bioinspired, bioinspiration, bio-inspired, bio-inspiration, bio inspired, bio inspiration, evolutionary, and swarm, as well its synonyms and variations. The ASEs were chosen according to Navau et al. (2013), which represent the most relevant for Computer Science. The ASEs selected were Web of Knowledge (ISIWoS), SCOPUS and IEEEXplore. The following boolean composition was used to perform the search: (FACES OR FACE OR FACIAL) AND (RECOGNITION OR DETECTION) AND (BIO-INSPIR* OR "BIO INSPIR*" OR BIOINSPIR* OR EVOLUTIONARY OR SWARM). However, as each ASE has its own search mechanism, this boolean query suffered some changes preserving its semantic meaning.

\subsection{Conducting the Review}

After defining the search engines and the boolean query, closure requirements are necessary, such as objective, inclusion, and exclusion criteria, which are presented as follows:

- Objective Criteria (OC)

- Year range : 2012 - 2018

- Document type : Articles

- Language : English

- Availability : Any

- Completeness : Full or short

- Duplicates : Yes
- Inclusion Criteria (IC)

- $\mathrm{IC}_{1}$ : To include works if, and only if it uses any swarm intelligence and/or evolutionary algorithms applied to FR systems.

- Exclusion Criteria (EX)

- $\mathrm{EX}_{1}$ : Remove works based on video recognition.

- $\mathrm{EX}_{2}$ : Remove works which aims to recognize only pose or emotions.

- $\mathrm{EX}_{3}$ : Remove works which use thermal face images.

- $\mathrm{EX}_{4}$ : Remove works which fuses features from other biometric techniques with face features.

- $\mathrm{EX}_{5}$ : Remove works which uses 3D face features.

- $\mathrm{EX}_{6}$ : Remove duplicated works.

Based on the above rules, it was established that if a work fits into any EX or does not fit into any OC, then it should be excluded. The evaluation of each paper returned by the boolean query was done in this order: OC, EX, and IC. Figure 1 shows the relevant works found for each ASE.

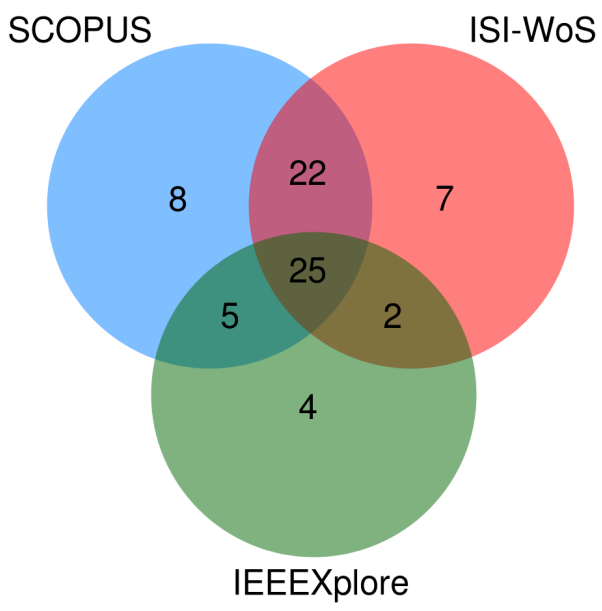

Figure 1: Relevant works found for each ASE.

After removing the duplicated works, about seventy three papers are kept for analysis.

\section{Face Recognition}

Traditional security systems based on encryption and passwords have proven vulnerable and easily breakable. Hence, the biometric technology became crucial for many domains (Nebti and Boukerram; 2017). Among them, 2D FR systems do not require any user interaction which becomes an advantage compared with other biometric technologies (Kim et al.; 2016). However, some issues arise on image acquisition such as occlusion, different pose and expressions and illumination variation. In this context, much interest and research have been focused on the field of FR and consequently, an increased number of bio-inspired FR systems had been emerged for different purposes which include feature selection, parameters optimization, template matching and classification. 
According to Rao and Rao (2016), the feature selection technique in a FR system consists in extracting the best features subset from the original images dataset, aiming to improve recognition rate. Experimental results have proven to compensate illumination and expression variations. Many authors employed bio-inspired algorithms to optimize the intrinsic parameters in their proposed methodologies, such as selecting the parameters $\mathrm{G}$ and C of Support Vector Machine (SVM) classification (Valuvanathorn et al.; 2012), searching the optimum Hidden Markov Model (HMM) states and parameters (Farag et al.; 2016), or adjusting the parameters of a homomorphic filter (Plichoski et al.; 2017). In addition to these, there are other bio-inspired approaches that are employed for template matching, which consists in finding areas of an image that better match to a template image (Chidambaram et al.; 2014). As preprocessing step, template matching might deal with problems such as scale and rotation variations. Finally works were found for classification purposes, such as Nebti and Boukerram (2017) that employed a bio-inspired approach to classify a decision tree recursively until obtaining only one class representing the input face image, thus addressing illumination, pose and facial expression variations. Table 1 presents the acronyms used in this review to indicate in which specific problem, bio-inspired algorithms are employed in FR systems.

Table 1: Acronyms for the applications employed

\begin{tabular}{lr}
\hline \multicolumn{1}{c}{ Application } & Acronym \\
\hline Feature Selection & FS \\
Parameter Optimization & PO \\
Classification & CL \\
Template Matching & TM \\
\hline
\end{tabular}

\section{Bio-inspired Algorithms}

Bio-inspired algorithms may be classified into five different groups based on their source of inspiration, which are physico-chemical, Neural Networks, Immunological Systems, Swarm Intelligence (SI), and Evolutionary Computation (EC) according to Fister et al. (2013). EC and SI algorithms are the focus of this review due to the large amount of applications in 2D Face Recognition. Besides that, the application of Neural Networks in FR systems has brought attention of the research community by emerging a significant number of works. Therefore, we believe that they should have their own review on application of Neural Networks.

EC is based on the natural selection theory proposed by Darwin. Individuals of a population compete to survive in which the more adapted ones with higher reproductive chances will survive. Genetic Algorithms (GA) (Holland; 1973), Differential Evolution (DE) (Storn and Price; 1997), Genetic Programming (GP) (Koza; 1992), and Memetic Algorithm (MA) (Dawkins; 2016) are some EC-based algorithms that can be found in the literature related to 2D FR systems.

SI algorithms are inspired by the social and collective behavior of insects, such as ants, termites, bees, flock of birds and fish school. The collective and self-organized behavior that appears from local interactions is the intelligence found in those systems, which is actually called emergent behavior. Ant Colony Optimization (ACO) (Dorigo and Stutzle; 2003), Artificial Bee Colony (ABC) (Karaboga; 2005), Bacterial Foraging Optimization (BFO) (Passino; 2002), Bees Algorithm (BA) (Pham et al.; 2011), Cuckoo Search Algorithm (CSA) (Yang and Deb; 2009), Gravitational Search Algorithm (GSA) (Rashedi et al.; 2009), and Particle Swarm Optimization (PSO) (Eberhart and Shi; 2011) are some SI-based algorithms found in the literature that are applied to 2D FR systems.

In the next section, each algorithm is briefly detailed followed by their respective related works in the field of 2D FR. Displayed in alphabetical order, the first two algorithms belong to EC and the subsequent seven algorithms belong to SI. Also, for each algorithm, the works reviewed are grouped according to their specific application following the acronyms order presented in Table 1.

\subsection{Differential Evolution}

Differential Evolution (DE) was proposed by Storn and Price (1997). This algorithm initializes with randomly generated individuals in the search space. New individuals are generated adding the weighted difference between individuals to a third one, namely target. This routine is called mutation. Then, the target individual is combined with a randomly preselected individual resulting the trial individual and represents crossover in DE. If the fitness of the trial individual is worse than the target's fitness value, the trial is discarded. Otherwise, the trial individual replaces the target individual in the next generation, and represents greedy selection. This process repeats until a stop criteria is reached.

In our research for $\mathrm{DE}$ applications, four works found in the literature using FS. But, among these, three works also employed for PO. Mallipeddi and Lee (2012) used the DE algorithm in their system to select the optimal Principal Component Analysis (PCA) features. In DE, each population member encodes the index of $d$ features to be selected from the amount of $n$ features, and the search is guided by maximizing the distance between classes. To validate their methodology, Yale, Yale B, ORL, and AR databases were used. Oh et al. (2013) used DE to optimize the parameters of a Radial Basis Function Neural Network (RBFNN) and also for feature selection of combined PCA and Linear Discriminant Analysis (LDA) features. The DE is represented as a vector containing the learning rate, momentum coefficient, and fuzzification coefficient parameters, as well as the selected feature subset. In a later experiment, they used only the 2D-LDA features on the Yale and ORL databases (Yoo et al.; 2013). Recently, they performed a comparative study of feature extraction methods and their application to RBFNN using the same FR systems architecture but with $2 \mathrm{D}^{2} \mathrm{LDA}$ features (Oh et al.; 2016). 


\subsection{Genetic Algorithm}

The Genetic Algorithm was proposed by Holland (1973), and it is inspired by Darwin's evolution theory. The concepts of evolution and natural selection is used to guide the search for better solutions in a problem search space. The crossover routine simulates reproduction combining two individuals previously selected to generate a new one, then, the mutation operator is applied at each newly generated individual. It is usually represented as binary strings, so they can be decoded to almost any desired representation.

From this survey, we found three works employing the GA for feature selection (FS). Vignolo et al. (2012) proposed the use of GA to select the features extracted by means of Active Shape Model (ASM). The search is guided by a classifier so that the classification success rate is assigned as fitness value for each evaluated individual. The experiments were performed using University of ESSEX face Recognition Data. In another work, Vignolo et al. (2013) also used ASM for extracting features, but with a modified version of GA called Multi-Objective Genetic Algorithm (MOGA), in which the rank of an individual is the number of chromosomes in the population by which it dominates. Also, the author proposed an aggregative fitness function, which combines classification accuracy and the number of features in a single equation. Loderer and Pavlovičová (2014) proposed to optimize the parameters of Local Binary Patterns (LBP) such as type of pattern, size of blocks, distance measure, and the dimension of histograms using GA. The chromosome is represented as a sequence of values which will be optimized. To validate their methodology CMU-PIE, Yale B, and ORL databases were used.

\subsection{Artificial Bee Colony}

The Artificial Bee Colony (ABC) algorithm was proposed by Karaboga (2005). The inspiration comes from the natural foraging behavior of honey bees to find the optimal food source. Bees estimate the location of the food source by measuring the amount of energy spent as they fly, as well as the direction. The location and quality of the food source is shared with their nest-mates by performing a waggle dance and trophallaxis (direct contact). In the $\mathrm{ABC}$, bees aim to discover places of food sources (solutions) with high amount of nectar, that represents a good solution. Many aspects of the bees and other insects have been explained by the principle of selforganization (Eberhart and Shi; 2011). Foraging behavior for food sources depends on the three types of bees in the colony: the scout that randomly flies in the search space for new food sources, the employed bee that exploits the neighborhood of their locations selecting a random solution to perturb (new food source), and the onlooker bee (information obtained from waggle dance) that uses the population fitness to probabilistically select a guiding solution to exploit its neighborhood. If a new source is better than the previous one a greedy selection strategy is employed to determine the new food source.

In the literature, according to our research scope, three works are found applying the $A B C$ algorithm in 2D FR systems in which two for FS and one for TM. Chakrabarty et al. (2013) proposed the use of a Lévy-mutated $A B C$ algorithm to derive optimal Volterra kernels simultaneously maximizing interclass distances and minimizing intra-class distances in the feature space. The performance of the proposed methodology was validated in Yale A and Yale B databases. Khan and Gupta (2016) applied the ABC to reduce the number of sub-windows in an extraction algorithm. In this work, the bees select a subwindow and the solution is evaluated by the subwindow average. Experiments were carried out on University of ESSEX face Recognition Data and VITM datasets. In addition the previous two works, Chidambaram et al. (2014) proposed a multiple face recognition approach using an improved $A B C$ algorithm to search local features extracted by Speeded Up Robust Features (SURF). The individual is represented as a four-dimensional vector containing horizontal and vertical coordinates, scale factor and rotation angle, then an image patch is cut from the still image and its interest points are identified for matching with the target face image. The distance measures between the images were used to guide the search. Experiments were carried out on BIO-INFO database.

\subsection{Ant Colony Optimization}

Ant Colony Optimization (ACO) was proposed by Dorigo and Stutzle (2003). It's source of inspiration comes from the collective behavior of ants in finding the shortest path from the nest to the food source using the substance called pheromone. Ants drop pheromone on the ground as they travel and tends to follow the path with more pheromone, thus, after several trips to the food source the shortest path will retain more pheromone leading more ants to it.

Only one work using the ACO was found in this review and is an ensemble with $A B C$ algorithm, thus, it is described latter on Subsection 4.10.

\subsection{Bacterial Foraging Optimization}

The Bacterial Foraging Optimization (BFO) was proposed by Passino (2002) and it is inspired by the bacteria foraging behavior. The E. Coli bacterium move itself rotating its flagella around their body. If rotates counterclockwise, they propel the bacterium along a trajectory (run or swim), otherwise, they pull on the bacterium in different directions (tumble). Alternating properly between these two modes, it keeps bacteria in places with higher concentration of nutrients. In BFO algorithm, the bacteria represent the solutions, and its health, the quality of the solution. At first, bacteria tumble and swim (chemotaxis) in the search space, and then, the half of the population are killed based on the their health and the other half are duplicated (reproduction). After a certain number of reproduction steps, some bacteria are probabilistically chosen to be killed, and new ones are randomly generated (elimination-dispersal).

In this section, two relevant works can be mentioned that employed BFO algorithm focusing on FS and PO. The first work was proposed by Panda and Naik (2015) which is a modified version of BFO called 
Adaptive Crossover Bacterial Foraging Optimization Algorithm (ACBFOA) to find optimal subset of features reduced by LDA and PCA. In ACBFOA, the algorithm incorporates adaptive chemotaxis and also inherits the crossover mechanism of genetic algorithm. Experiments were performed on Color FERET, Yale A and UMIST data sets. Meanwhile the second work was proposed by Yadav et al. (2013) that mitigated the effect of facial changes by combining the global features of LBP and local facial regions at match score level by means of the BFO algorithm. The objective function is defined for learning the weights to be employed in a weighted sum rule fusion. Experimental results are presented using the FG-Net and IIITDelhi face aging databases.

\subsection{Bees Algorithm}

The Bees Algorithm (BA) was proposed by Pham et al. (2011), and it is inspired by the bees foraging behavior, as in the $A B C$ but with different internal routines. In this algorithm, a bee represents a possible solution, and a solution represents a visited site. The scout bees are randomly placed in the search space from which the bees with highest fitness will become selected bees and sites visited by them are chosen for local search.

Only one work using the BA was found in this review and it is developed with PSO algorithm. Hence it is described in Subsection 4.10 which specifically discusses about ensembled approaches.

\subsection{Cuckoo Search Algorithm}

The Cuckoo Search Algorithm (CSA) was proposed by Yang and Deb (2009), and it is inspired by the brood parasitic behavior of some cuckoo species, in combination with the Lévy flight behavior. These cuckoos lay their eggs in communal nests for other bird species to hatch. The CSA is based in three main rules: 1) Each cuckoo lays one egg at a time, and dump its egg in a randomly chosen nest; 2) The best nests with high quality of eggs will be carried over to the next generations; 3) The number of available host nests is fixed and the egg laid by a cuckoo is discovered by the host bird with a probability. In this case, the host bird can either throw the egg away or abandon the nest and build a completely new nest. In the proposed algorithm, each egg in a nest represents a solution and a cuckoo egg represent a new solution. The aim is to use the new and potentially better solutions (cuckoos) to replace a not-so-good solution in the nests.

Only one work was found in literature that employed CS algorithm for FS. Naik and Panda (2016) proposed an adaptive version of CSA (ACS) to find the optimal feature vectors for classification on Intrinsic Discriminant Analysis (ICA) feature space. In ACS, the step size is made adaptive from the knowledge of its fitness function value and its current position in the search space. For performance analysis, the YALE, ORL, and Color FERET databases were used.

\subsection{Gravitational Search Algorithm}

The Gravitational Search Algorithm (GSA) was proposed by Rashedi et al. (2009). This algorithm is inspired by the law of gravity and mass interactions in which particles are considered as objects and their performance is measured by their masses. All these objects attract each other causing their movement in the search space. When a solution is better with heavy mass, then it naturally moves more slowly than lighter ones, and thus, leading to more local search. The main difference of GSA from PSO is the local communication between objects that uses a gravitational factor.

During the SLR process about GSA, only one work was found for FS. Chakraborti and Chatterjee (2014) proposed to use a binary variation of GSA with dynamic adaptive inertia weight (BAW-GSA) to select the relevant features extracted by LBP, Modified Census Transform (MCT) and Local Gradient Pattern (LGP). The fitness function was implemented as the ratio of the within class distance (Intra-Class) to the between class distance (Inter-Class). The experiments were carried out on Yale A, Yale B, ORL, LFW and AR databases.

\subsection{Particle Swarm Optimization}

Particle Swarm Optimization (PSO) was proposed by Eberhart and Shi (2011) in 1995. The PSO algorithm is inspired by the coordinated behavior of a flock of birds or a fish school. The search mechanism is based on the acceleration of the particles, being attracted by the global best position (social component) and the personal best position (cognitive component) found so far. The algorithm initiates with a random population of particles with it's own velocities, which are responsible for moving the particles around the search space. At each iteration the velocities are updated until reaches a predefined stop criteria. It's canonical version were used for continuous optimization problems, however nowadays we may find in literature several other adaptations for different types of optimization problems e.g. discrete optimization problems (Kennedy and Eberhart; 1997). Among a wide range of works in which the PSO algorithm is used, we attempt to describe in the present section the forty three works that are developed for FS followed by eleven works that used for PO.

According to the time range established for this review, most works found have used BPSO for FS, guiding the search through inter-class maximization and intra-class minimization distances on Discrete Wavelet Transform (DWT) feature space. Beside classical implementations of DWT (Surabhi et al.; 2012; Yaji et al.; 2012; Rinky et al.; 2012; Roohi et al.; 2013; Prabhu et al.; 2013; Niveditha et al.; 2015), other versions were employed, such as Threshold based DWT (Vidya et al.; 2012; Murthy et al.; 2012), Raster Scan Discrete Wavelet Transform (RDWT) (Khashu et al.; 2014) and a hybrid approach using Thresholded Wavelet Edge Enhancement Transform (TWEET) and DWT (Hegade et al.; 2013). Methods employing modified versions of BPSO were proposed, such as Threshold Binary Particle Swarm Optimization (ThBPSO) (Krisshna et al.; 2014). In ThPSO, 
the recurrence of selected features is considered based on a threshold set by the user. Sattiraju et al. (2013) proposed to use a modified version of BPSO called Adaptive Multi-Level Threshold Binary Particle Swarm Optimization (AMBPSO) to select features extracted by DWT. In AMBPSO, the 10 best global solutions are stored in a matrix and are used to guide the search. In addition to the works mentioned previously, Khadhraoui et al. (2016) used a deterministic parameter control technique on BPSO based on the number of iterations differing from traditional implementations. The benchmark databases mostly used to validate these methodologies are presented in descending order: Color FERET, Yale B, ORL, CMU-PIE, UMIST, PHPD and FEI.

A couple of works used Discrete Cosine Transform (DCT) for feature extraction and BPSO for selecting the most discriminant features based on interclass maximization and intra-class minimization distances (Divya et al.; 2012; Gagan et al.; 2012), moreover an accelerated version of BPSO (ABPSO) was employed on DCT feature space (Aneesh et al.; 2012). In ABPSO, the velocity is updated for each iteration by summing it with the previous positional values for each particle. Ensemble approaches were experimented with DCT, such as Deepa et al. (2012) that used BPSO to select features extracted by DFT and DCT, and Ajaya et al. (2014) proposed an approach to select extracted features from Contourlet Transform (CT) and DCT. On CT feature space, PCA was employed for dimensionality reduction and BPSO for FS. Last, (Darestani et al.; 2013) extracted features using only CT and employed BPSO for feature selection. Then, in the classification stage, a classifier based on a artificial neural network was used with PSOoptimized hidden layer size and learning rate. In the works mention here, the mostly used databases are presented in descending order: ORL, Color FERET, Yale B, UMIST, CMU-PIE, PHPD, JAFFE and CASPERL.

A considerable number of works using DWT and DCT along with PSO were also performed (D'Cunha et al.; 2013; Nischal et al.; 2013; Rao et al.; 2014; Babu, Shreyas, Manikantan and Ramachandran; 2014; Kodandaram et al.; 2015). Also, Soumya et al. (2013) proposed a approach using BPSO to select features extracted from DWT and DCT. However, besides class separation, the authors used DCT trace in the fitness relation. Similarly Rao and Rao (2016) used BPSO for FS extracted from DWT and Slope-form Triangular Discrete Cosine Transform (STDCT). In this work, fitness function was based in preserving maximum precision in order to represent the original feature set. The works mentioned in this paragraph used the following databases which are in descending order: Color FERET, CMU-PIE, PHPD, Yale B, UMIST, ORL, FEI, GT, IFD and JAFFE.

Other approaches for FS using BPSO and distance between classes were also proposed, such as the work developed by Shanbhag et al. (2014). They developed the work based on BPSO to search the features extracted by Wavelet Transform Feature Extraction (WTFE) for the optimal subset. Shetty et al. (2013) proposed a similar work, however, to select features extracted by Stationary Wavelet Transform (SWT) based technique namely Shift
Invariance based Feature Extraction (SIFE). The authors claimed that they have used a modified version of BPSO called Weighted Binary Particle Swarm Optimizer (WBPSO), but, actually, they just considered the number of times a particular feature is being selected in the fitness computation. Also, Babu, Birajdhar and Tambad (2014) used the recurrence of a feature in fitness evaluation and called Conservative BPSO to select features from SWT feature space. Abhishree et al. (2015) proposed to use BPSO to select features extracted by Gabor filter technique. The same way, Kishore et al. (2014) used Gabor filter, but combined with Fast Fourier Transform (FFT). Differently, Cheng et al. (2014) searched for the optimal subset on the Self Quotient Image (SQI) features space meanwhile Nema and Thakur (2015) proposed to select features extracted by LDA. In their work the algorithm was adapted with a deterministic parameter control technique which decreases $C 1$ and increases $C_{2}$ exponentially with time. Like others, Vora et al. (2014) tried the FS on Gamma Ray Burst Rhombus Star (GRBRS) feature mask space and Varun et al. (2015) on Block-wise Hough Transform (HT) feature space. The final work which used the distance between class as a fitness evaluation was proposed by Varadarajan et al. (2015). In this work, the author tested a modified version of BPSO called Exponential Binary Particle Swarm Optimization (EBPSO) to select the features extracted using Block based Additive Fusion. The group of articles mentioned in the present paragraph used the databases: Color FERET, CMU-PIE, Yale B, PHPD, FEI, ORL, IFD, LFW, GT and CAS-PERL.

Different approaches for FS using PSO and its variants were employed, such as Lei et al. (2012) who proposed to use a variation of PSO denominated as Fast Static Particle Swarm Optimization (FSPSO). It treats the whole initial feature set as a static particle swarm in which no new particle would be generated in high dimensional space, and the proposed method takes filter and wrapper strategy to pick out the most discriminative feature particle subset. In the universe of FS works, Shieh et al. (2014) proposed to use PSO to select features extracted by PCA using SVM as fitness function. Yin, Qiao, Fu and Xia (2014) proposed to use BPSO for feature selection on DCT coefficients feature space.In this approach, the fitness function is based on classification rate and dimensionality. In another work, YIN, FU and SUN (2014) used the same approach, but, they reduced the search space for BPSO algorithm by preselecting DCT coefficients according to a separability criterion. In addition to the modified versions of PSO, it is becoming common the use of modified versions BPSO. As an example, we can mention Sah et al. (2015) who proposed a modified version of BPSO called Logarithmic Binary Particle Swarm Optimization (LBPSO) to select features extracted by Entropic Gabor Wavelet Transform (GWT). In LBPSO, the global solution and particle best positions are weighted instead of their current positions. The feature vector is optimized by sampling the vector and choosing the one with the highest entropy. Among the works that we have mentioned, the work proposed by Mollaee and Moattar (2016) is somehow different since they proposed to use the PSO algorithm to aid discriminant ICA in finding multivariate data 
with lower dimension and independent features by maximizing Negentropy, as well as Fisher criterion. Zhang and Peng (2016) proposed to use PSO to find out the optimum combination of the basis and variety from a basis plus variety model on a high-dimensional unit sphere in terms of the minimum L2 distance relative to the query image. In their model, the query image is approximately a linear combination of the basis image and variety image. The basis images are the neutral images of subjects and variety images are generalized from multi-sample subjects. The basis of the optimum point gives the identity information for classification. The databases used by the works mentioned in this paragraph were: Yale B, ORL, Color FERET, CMU-PIE, $\mathrm{AR}$ and MIT.

The bio-inspired approaches for PO were also employed the PSO algorithms. Here, we comment about the works related to SVM parameters optimization. Valuvanathorn et al. (2012) developed the work to aid SVM classification by selecting the parameters $\mathrm{G}$ and $\mathrm{C}$ automatically (PSO-SVM). In the next work, a modified version of PSO, called Opposition Particle Swarm Optimization (OPSO) was presented by Hasan et al. (2013) to optimize the SVM parameters in training and testing features extracted by PCA. In OPSO, two populations are generated: the first one is random and the second is opposition population which is based on the first population values. In this work, similar to others, Xiao et al. (2014) used PSO combined with grid-search to optimize the parameters of a Radial Basis Function (RBF) kernel in SVM. Similarly, Zou and Zhang (2016) presented their PO work using the recognition rate to calculate the fitness of each particle. The above mentioned works in the present paragraph used mostly the following databases: ORL, Color FERET, Yale A and BioID.

Besides optimizing SVM parameters, some works that optimize other parameters were also found, such as Pan et al. (2013) who proposed to replace exhaustive search used in Adaboost framework with PSO. The authors claimed that PSO is used as FS procedure, but as each particle is encoded with a feature parameter set (type, $x_{s}, y_{s}, \theta$, width, height, sampling_points, radius). The fitness function was defined as the normalized classification error rate. Banerjee and Datta (2013) proposed to use PSO for parameters optimization for both constrained and unconstrained type in which particle vectors are considered as correlated parameters to be optimized. The false acceptance rate was used as objective function to be minimized. Trinh et al. (2014) proposed to use PSO algorithm to find optimal weights to fuse global and local Fourier-Mellin Transform (FMT) features at score-level. The fitness function is evaluated by calculating the recognition rate related to a specific set of weights. FernándezMartínez and Cernea (2015) proposed to use a modified version of BPSO called RR-PSO to optimize the parameters of SCAV1. SCAV1 is a supervised ensemble learning algorithm based on six nearestneighbor classifiers based on histogram, variogram, texture analysis, edges, DWT and Zernike moments. In RR-PSO, global and local search are balanced by adopting regressive discretization in acceleration and in velocity of the PSO continuous model. Farag et al. (2016) proposed to apply PSO to search the optimum HMM states and parameters. In their approach, maximum accuracy and minimum feature dimension was used to guide the search. Kim et al. (2016) proposed to use PSO algorithm to optimize the parameters of RBFNN such as the number of nodes and fuzzification coefficient. The classification rate is used as the fitness value. Finally, Plichoski et al. (2017) proposed to use PSO to optimize Homomorphic filter (HF) parameters namely high and low frequency factors, cut-off frequency and filter's order. The recognition rate is used as fitness function to guide the algorithms search. The most used database by the presented works were: ORL, CMU-PIE, Yale A, PHPD, MIT, AR, Korean Face Database, PUT, UCSD and IC\&CI.

\subsection{Ensemble-based approaches}

This section refers to applications of ensemble bio-inspired approaches in which more than one optimization algorithm is used in the same FR system. In our review, some hybrid approaches were found in which the first work embedded the algorithms in their system for FS and CL. In the other works reviewed, bio-inspired approaches were employed for FS, followed by PO and CL respectively.

Kaur et al. (2013b) used ABC to select the features extracted by DWT, wherein optimization is driven by terms of correlation value for pattern recognition. For CL application task, ACO is used by measuring the distances between the selected features. The authors used their own image sets to test their methodology. Khan et al. (2015) proposed to use BPSO along with GA algorithm for feature selection. Global and local features were extracted from the image using DCT and LBP, respectively. The system was evaluated using ORL and LFW face databases. Kallianpur et al. (2016) proposed to use a modified discrete version of ABC called Hybrid-Discrete Artificial Bee Colony algorithm (H-DABC) to select features extracted from DWT. This ABC version contains certain elements from PSO, then, its an hybrid algorithm. Their methodology was validated using LFW, Color FERET and ORL. Dora et al. (2017) proposed to use a hybrid algorithm of PSO and GSA (PSO-GSA) for optimizing the parameters of an evolutionary single Gabor kernel (ESGK) filter which is used for feature extraction. The fitness function used for selecting optimal filter is the Gabor energy vector. The experiments were performed on Color FERET, ORL, UMIST, GT and LFW databases. The last work reviewed is proposed by Nebti and Boukerram (2017) using BA and a decision tree based PSO as classifiers. The tree is represented by the classes, then BPSO classifies them recursively until obtaining only one class representing the input face image. BA searches for the best training faces which are the most similar to the faces being recognized. The fitness function for both algorithms is the sum of the Euclidean distances between the current particle and the testing sample features. The two are combined with a decision tree based fuzzy SVM using majority vote. The experiments were conducted on ORL, YALE, FERET and UMIST. 


\section{Summary and Discussions}

As seen in Section 4, most of the bio-inspired algorithms in 2D FR systems have been applied to FS, PO, CL and TM. Seventy three scientific articles were analyzed focusing on the problem approached, which bio-inspired algorithm are used, how candidate solutions are represented, how fitness function is modeled, and what databases are employed.

Figure 2 presents the distribution of bio-inspired algorithms among the analyzed works. A huge gap between the use of the PSO algorithm $(73.41 \%)$ in comparison to other algorithms $(26.59 \%)$ can be noticed. Some reasons for the popularity of PSO algorithm in 2D FR systems might be because of its good performance, its implementation simplicity, and the use of few parameters to be tuned. Also, as pointed out by Alsalibi et al. (2015), PSO algorithm requires less training time, has good scalability and high convergence rate. According to an extensive review done by Zhang et al. (2015), the number of publications related to PSO is the highest among others reaching around 1,000 per year. However, the few works developed using other bio-inspired algorithms provides room for exploiting their features.

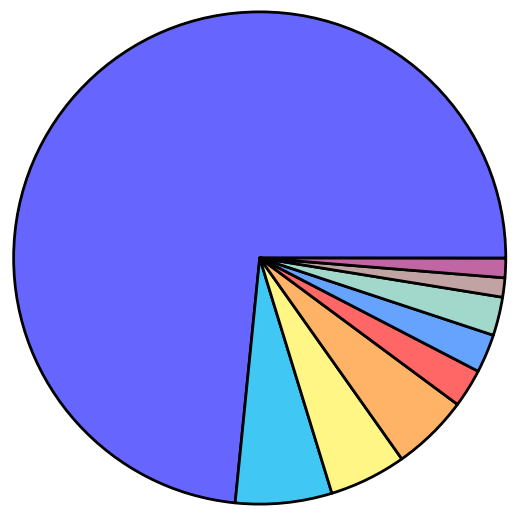

PSO-73.41\%

$\square \mathrm{ABC}-6.33 \%$

$\square \mathrm{DE}-5.06 \%$

GA- $5.06 \%$

GSA-2.53\%

BA-2.53\%

BFO-2.53\%

ACO-1.265\%

$\mathrm{CS}-1.265 \%$

Figure 2: Bio-inspired algorithms distribution in relation to the analyzed works

Among the four applications employed, the FS applications reaches the highest rate of $76.315 \%$, followed by PO with $19.74 \%$. In our review, we found only two works for classification and one work for template matching. Figure 3 shows the distribution of applications per algorithm in which the dominance of FS applications can be clearly observed. The high usage of FS can be justified since its use as a dimensional reduction approach becomes attractive and sometimes required. Additionally most of the works that deals with images and videos requires FS as an important step and it is an important component of many pattern recognition tasks with very high-dimensional data (Gui et al.; 2017).

For every work applied for FS, the candidate solution was represented as a vector containing the selected features. Also, in most works the search was driven aiming to maximize the distance between classes. The most used feature extraction techniques are presented in Figure 4. The DWT technique is the most employed $(41.82 \%)$ mainly because of its

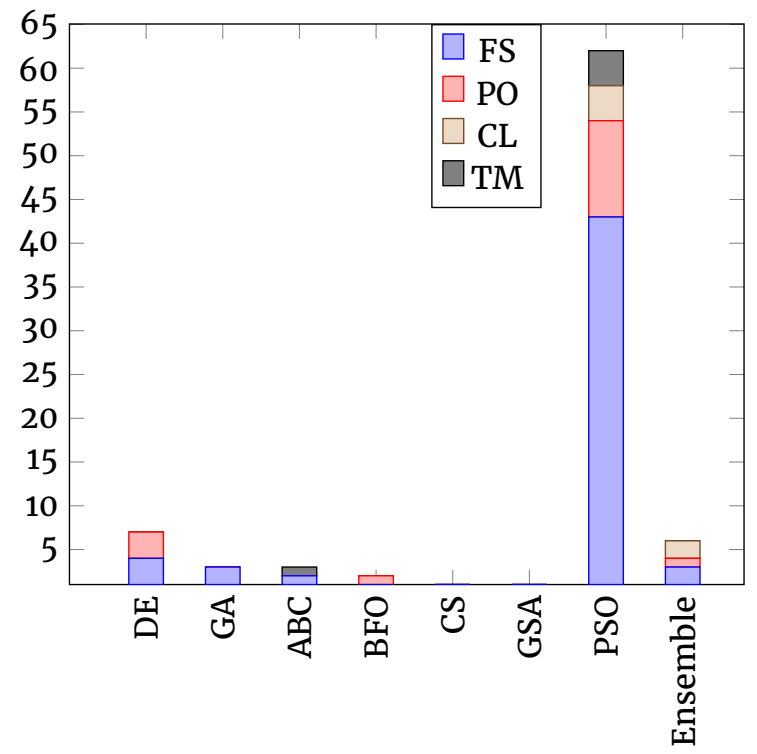

Figure 3: Distribution of applications per algorithm

good performance. Briefly, the DWT has the ability to provide a multi-resolution analysis of the image and the capability of decomposing the image into sub-bands in both time and frequency domains producing detailed information and coarse grained approximations (Du and Ward; 2005). Also, a number of works employing the DCT technique is expressive (27.27\%). According to Rao and Rao (2016), the DCT is a popular transformation technique based on the energy compaction property, wherein the information or features tend to be concentrated in low frequency components or at the corner of the spectrum. Regarding the dimensionality reduction techniques, the LDA and PCA are popular in 2D FR systems with $9.09 \%$ and $7.27 \%$, participation respectively. The other techniques represent in average about $3.6 \%$ each. For the PO task, the candidate solution is represented as the parameters itself and in $56.25 \%$ of the works, the fitness function is constructed based on accuracy (recognition rate), followed by distance between classes maximization $(18.75 \%)$. The most used techniques for tuning were the SVM $(26.66 \%)$, the RBFNN (20\%) and the LBP $(13.33 \%)$.

For the CL task, distinct solution representations are employed. For example, one work used the image pixels as solution representation (Kaur et al.; 2013b) and another used the database classes (Nebti and Boukerram; 2017). For the TM task, the individual is represented as a four-dimensional vector containing horizontal and vertical coordinates, scale factor and rotation angle. All works used the Euclidean distance measure to drive the search and to calculate the distance between the images (Chidambaram et al.; 2014).

Figure 5 shows the distribution of most used bioinspired algorithms per year. The number of works employing the PSO far exceeds all other algorithms. However, the $\mathrm{ABC}$ and the set $\mathrm{DE}$ and GA occupy the second and third rankings respectively.

When analyzing the works presented in this review, we realize that the experimental methodology 


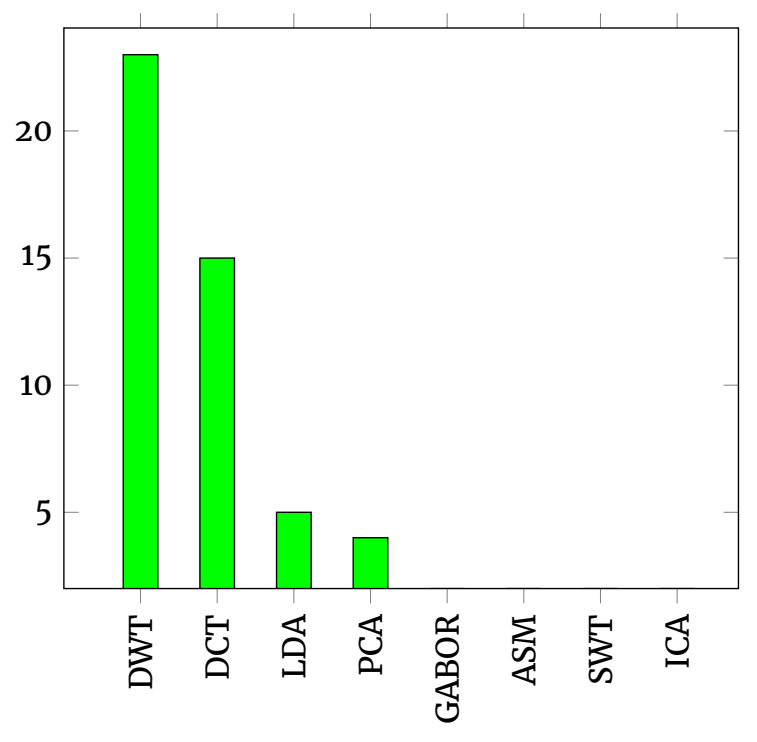

Figure 4: Most used feature extraction techniques

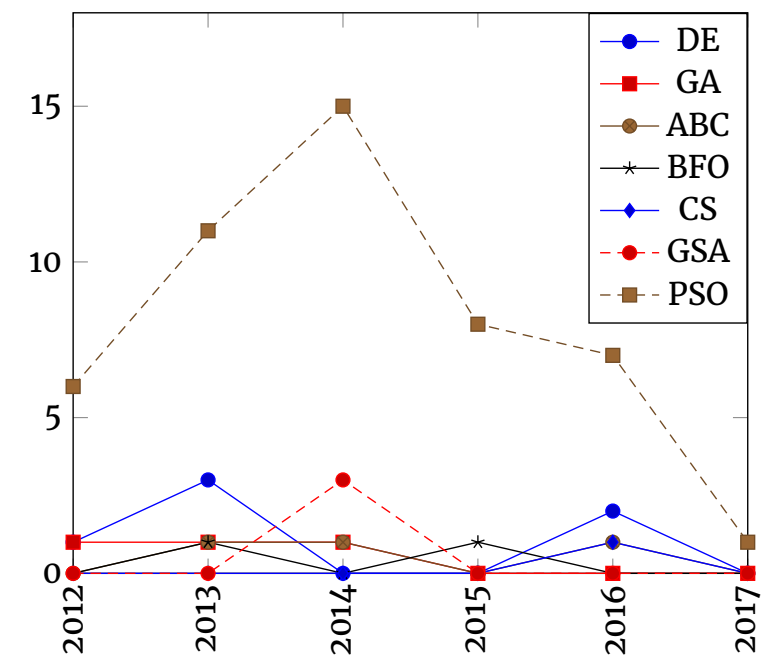

Figure 5: Number of bio-inspired algorithms employed per year

performed by each author usually differs, which sometimes makes it unreliable, difficult to compare with others and to replicate results. As an example, we can mention the use of the ORL database which contains 400 images of 40 individuals with 10 images of each one varying pose. Khadhraoui et al. (2016) used the first 5 images per person of all subjects for training and the remaining 5 images per person of all subjects for testing. On the other hand, from the same database Fernández-Martínez and Cernea (2015) used 200 images for training, 160 images for testing and 40 images to perform blind validation. Despite that, we investigated the most used benchmark databases and are presented in Table 2. The complexity of the databases is defined by its size and categories such as pose, illumination, expressions and occlusion. The most used database is the FERET (representing $20.21 \%$ of the works) which contains 14,126 images with $512 \times 768$ pixels and 1,199 individuals varying pose, illumination and expressions Alsalibi et al. (2015). The next position is occupied by the ORL database with $17.55 \%$ and Yale B with $13.83 \%$ of the works. Yale B database is a well known database because of its several degrees of illumination and its cropped version of face images which is important to investigate the illumination variation problem. The remaining databases information are shown in references described in Table 2.

\section{Conclusion and Future Work}

Nowadays, in different parts of the society, we can find a pool of $2 \mathrm{D}$ FR systems that perform well under controlled settings. However, real-time situations require systems that should deal with uncontrolled settings and thus creating an open research gap. The major challenges found in the $2 \mathrm{D}$ FR systems refers to the high-dimensionality of the dataset, a wide range of image variations and the useless information in the images leading to misclassification. Such complexity makes necessary the use of optimization methods as bio-inspired algorithms to construct robust FR systems. Hence, the researchers from the whole world have been proposed many 2D FR approaches using bio-inspired algorithms. Hence, in this context, to map these approaches we proposed the present Systematic Literature Mapping.

As discussed in this review, bio-inspired algorithms have being applied in many tasks such as template matching, classification, feature selection, and parameters optimization. The last two applications, FS and PO, are the most used to improve 2D FR systems with $76.135 \%$ and $19.74 \%$ of the works, respectively. Among different bio-inspired algorithms discussed in this work, the use of PSO far exceeds the other algorithms and it is present in $73.41 \%$ of the analyzed works. The reasons for this might be due to its good performance, simplicity to implement and few parameters to tune. Furthermore, it is possible to note that bio-inspired 2D FR systems are trending towards the PSO algorithm which suggests a reliable choice for future applications, as well as the usage of the DWT technique for feature extraction and SVM for classification. A complete exposure of the most used databases is also presented in this work. Among them the top three are the FERET, the ORL and the Yale B databases. The possible future works can be conducted in analyzing the performance of the bio-inspired 2D FR systems.

\section{References}

Abate, A. F., Nappi, M., Riccio, D. and Sabatino, G. (2007). 2d and 3d face recognition: A survey, Pattern recognition letters 28(14): 1885-1906.

Abhishree, T., Latha, J., Manikantan, K. and Ramachandran, S. (2015). Face recognition using gabor filter based feature extraction with anisotropic diffusion as a pre-processing technique, procedia Computer Science 45: 312-321.

Ajaya, H., Gowda, M. E., Manikantan, K. and Ramachandran, S. (2014). Face recognition by optimal band selection in contourlet transform 
Table 2: Most popular databases.

\begin{tabular}{|c|c|c|c|c|c|}
\hline Database & Subjects & Total Images & Variations & Reference & Occurrence (\%) \\
\hline Facial Recognition Technology (FERET) & 1,199 & 14,126 & $\mathrm{p}, \mathrm{i}, \mathrm{e}$ & Phillips et al. (2000) & 20.21 \\
\hline Olivetti Research Laboratory (ORL) & 40 & 400 & $\mathrm{p}$ & Samaria and Harter (1994) & 17.55 \\
\hline Yale B database & 10 & 5,760 & $\mathrm{p}, \mathrm{i}$ & Georghiades et al. (2001) & 13.83 \\
\hline Carnegie Mellon university pose, illumination, and expression (CMU PIE) & 68 & 4,136 & $\mathrm{p}, \mathrm{i}, \mathrm{e}$ & Gross et al. (2010) & 10.64 \\
\hline University of Manchester Institute of Science and Technology (UMIST) & 20 & 564 & $\mathrm{p}$ & Wechsler et al. (2012) & 6.38 \\
\hline Pointing Head and Pose Image Database (PHPD) & 15 & 2,790 & $\mathrm{p}, \mathrm{o}$ & Gourier et al. (2004) & 6.38 \\
\hline Yale A database & 15 & 165 & $\mathrm{i}, \mathrm{e}$ & Belhumeur et al. (1997) & 4.25 \\
\hline The FEI face database & 200 & 2,800 & $\mathrm{p}$ & Thomaz and Giraldi (2010) & 3.72 \\
\hline Labeled Faces in the Wild (LFW) & 1680 & $13,000+$ & $\mathrm{p}, \mathrm{i}, \mathrm{e}, \mathrm{o}$ & Huang et al. (2007) & 2.66 \\
\hline Aleix Martinez and Robert Benavente Face Database (AR) & 126 & 4,000 & $\mathrm{i}, \mathrm{e}, \mathrm{o}$ & Martinez (1998) & 2.13 \\
\hline $\begin{array}{l}\text { Indian Face Database (IFD) } \\
\text { If }\end{array}$ & 40 & 440 & e & Jain (2002) & 1.59 \\
\hline Georgia Tech (GT) Face Database & 50 & 750 & $\mathrm{p}, \mathrm{i}, \mathrm{e}$ & Chen et al. (2005) & 1.59 \\
\hline Academy of sciences pose, expression, accessories, and lighting (CAS-PEAL) & 1,040 & 30,900 & $\mathrm{p}, \mathrm{e}, \mathrm{i}, \mathrm{i}$ & Gao et al. (2008) & 1.06 \\
\hline Japanese Female Facial Expression (JAFFE) Database & 10 & 213 & e & Lyons et al. (1998) & 1.06 \\
\hline Massachusetts institute of technology - center for biological and computational learning (MIT-CBCL) & 10 & 2,000 & $\mathrm{p}, \mathrm{i}$ & Weyrauch et al. (2004) & 1.06 \\
\hline University of ESSEX face Recognition Data & 395 & 7,900 & $\mathrm{p}, \mathrm{i}, \mathrm{e}, \mathrm{e}$ & Spacek (2002) & 1.06 \\
\hline BIO-INFO & 138 & 276 & $\mathrm{i}$ & das Chagas Prodossimo et al. & 0.53 \\
\hline The BioID Face Database (BioID) & 23 & 1,521 & $\mathrm{p}, \mathrm{e}, \mathrm{o}$ & Jesorsky et al. (2001) & 0.53 \\
\hline Intelligent Control and Computational Intelligence Laboratory (IC\&CI) & 15 & 825 & $\mathrm{p}$ & Kim et al. (2016) & 0.53 \\
\hline Korean Face Database & 40 & 600 & $\mathrm{e}, \mathrm{i}$ & Trinh et al. (2014) & 0.53 \\
\hline $\begin{array}{l}\text { PUT Face Database } \\
\text { PUS }\end{array}$ & $\begin{array}{r}40 \\
100\end{array}$ & 9,971 & $\mathrm{p}, \mathrm{e}, \mathrm{o}$ & Kasinski et al. (2008) & $\begin{array}{l}0.53 \\
0.53\end{array}$ \\
\hline Honda/UCSD database & 20 & 560 & $\mathrm{p}$ & Kim et al. (2016) & 0.53 \\
\hline VITM College Gwalior Face Database & - & 200 & a & Khan and Gupta (2016) & 0.53 \\
\hline FG-Net & 82 & 1,002 & $\mathrm{a}$ & Khan and Gupta (2016) & 0.53 \\
\hline IIIDelphi & 102 & 2,618 & $\mathrm{p}, \mathrm{i}, \mathrm{e}$ & Yadav et al. (2013) & 0.53 \\
\hline
\end{tabular}

domain, Industrial and Information Systems (ICIIS), 2014 9th International Conference on, IEEE, pp. 1-8.

Alsalibi, B., Venkat, I., Subramanian, K., Lutfi, S. L. and Wilde, P. D. (2015). The impact of bio-inspired approaches toward the advancement of face recognition, ACM Computing Surveys (CSUR) 48(1): 5 .

Aneesh, M., Masand, A. A. and Manikantan, K. (2012). Optimal feature selection based on image pre-processing using accelerated binary particle swarm optimization for enhanced face recognition, Procedia Engineering 30: 750-758.

Babu, S. H., Birajdhar, S. A. and Tambad, S. (2014). Face recognition using entropy based face segregation as a pre-processing technique and conservative bpso based feature selection, Proceedings of the 2014 Indian Conference on Computer Vision Graphics and Image Processing, ACM, p. 46.

Babu, S. H., Shreyas, H., Manikantan, K. and Ramachandran, S. (2014). Face recognition using active illumination equalization and mirror image superposition as pre-processing techniques, Signal and Image Processing (ICSIP), 2014 Fifth International Conference on, IEEE, pp. 96-101.

Banerjee, P. K. and Datta, A. K. (2013). A preferential digital-optical correlator optimized by particle swarm technique for multi-class face recognition, Optics \& Laser Technology 50: 33-42.

Belhumeur, P. N., Hespanha, J. P. and Kriegman, D. J. (1997). Eigenfaces vs. fisherfaces: Recognition using class specific linear projection, IEEE Transactions on pattern analysis and machine intelligence 19(7): 711-720.

Bowyer, K. W., Chang, K. and Flynn, P. (2006). A survey of approaches and challenges in $3 \mathrm{~d}$ and multi-modal $3 \mathrm{~d}+2 \mathrm{~d}$ face recognition, Computer vision and image understanding 101(1): 1-15.

Chakrabarty, A., Jain, H. and Chatterjee, A. (2013). Volterra kernel based face recognition using artificial bee colonyoptimization, Engineering Applications of Artificial Intelligence 26(3): 1107-1114.

Chakraborti, T. and Chatterjee, A. (2014). A novel binary adaptive weight gsa based feature selection for face recognition using local gradient patterns, modified census transform, and local binary patterns, Engineering Applications of Artificial Intelligence 33: 80-90.

Chen, L., Man, H. and Nefian, A. V. (2005). Face recognition based on multi-class mapping of fisher scores, Pattern Recognition 38(6): 799-811.

Cheng, Y., Jin, Z., Hao, C. and Li, X. (2014). Illumination invariant face recognition with particle swarm optimization, Data Mining Workshop (ICDMW), 2014 IEEE International Conference on, IEEE, pp. 862-866.

Chidambaram, C., Marçal, M. S., Dorini, L. B., Neto, H. V. and Lopes, H. S. (2012). An improved abc algorithm approach using surf for face identification, International Conference on Intelligent Data Engineering and Automated Learning, Springer, pp. 143-150.

Chidambaram, C., Neto, H. V., Dorini, L. E. B. and Lopes, H. S. (2014). Multiple face recognition using local features and swarm intelligence, IEICE TRANSACTIONS on Information and Systems 97(6): 1614-1623.

Darestani, M. R. Y., Sheikhan, M. and Khademi, M. (2013). Face recognition using contourlet-based features and hybrid pso-neural model, Information and Knowledge Technology (IKT), 2013 5th Conference on, IEEE, pp. 1818-186.

das Chagas Prodossimo, F., Chidambaram, C., Hastreiter, R. L. F. and Lopes, H. S. (2012). Proposta de uma metodologia para a construç ao de um banco de imagens faciais normalizadas, PIE, Vol. 5 , p. 41368.

Dasgupta, D., Yu, S. and Nino, F. (2011). Recent advances in artificial immune systems: models and applications, Applied Soft Computing 11(2): 15741587.

Dawkins, R. (2016). The selfish gene, Oxford university press.

D'Cunha, N. W., Birajdhar, S. A., Manikantan, K. and Ramachandran, S. (2013). Face recognition using homomorphic filtering as a pre-processing technique, Emerging Trends in Communication, 
Control, Signal Processing \& Computing Applications (C2SPCA), 2013 International Conference on, IEEE, pp. 1-6.

De Jong, K. (2006). Evolutionary Computation: A Unified Approach, Bradford Book, Mit Press.

Deepa, G., Keerthi, R., Meghana, N. and Manikantan, K. (2012). Face recognition using spectrumbased feature extraction, Applied Soft Computing 12(9): 2913-2923.

Detroz, J. P., Hinz, M. and Hounsell, M. S. (2015). The use of literature review in informatics in education: a systematic mapping, Brazilian Journal of Computers in Education 23(01): 28.

Divya, R., Rath, A., Ramachandran, S. and Manikantan, K. (2012). Astroid shaped dct feature extraction for enhanced face recognition, Proceedings of the CUBE International Information Technology Conference, ACM, pp. 95-101.

Dora, L., Agrawal, S., Panda, R. and Abraham, A. (2017). An evolutionary single gabor kernel based filter approach to face recognition, Engineering Applications of Artificial Intelligence 62: 286-301.

Dorigo, M. and Stutzle, T. (2003). The ant colony optimization metaheuristic: Algorithms, applications, and advances, International series in operations research and management science pp. 251286.

Du, S. and Ward, R. (2005). Wavelet-based illumination normalization for face recognition, Image Processing, 2005. ICIP 2005. IEEE International Conference on, Vol. 2, IEEE, pp. II-954.

Eberhart, R. C. and Shi, Y. (2011). Computational intelligence: concepts to implementations, Elsevier.

Fan, X. and Verma, B. (2004). Face recognition: a new feature selection and classification technique, Proc. 7th Asia-Pacific Conference on Complex Systems.

Farag, M. M. M., Elghazaly, T. and Hefny, H. A. (2016). Face recognition system using hmmpso for feature selection, Computer Engineering Conference (ICENCO), 2016 12th International, IEEE, pp. 105-110.

Fernández-Martínez, J. L. and Cernea, A. (2015). Exploring the uncertainty space of ensemble classifiers in face recognition, International Journal of Pattern Recognition and Artificial Intelligence 29(03): 1556002.

Fister, Jr., I., Yang, X.-S., Fister, I., Brest, J. and Fister, D. (2013). A Brief Review of Nature-Inspired Algorithms for Optimization, ArXiv e-prints .

Gagan, R., Sanghavi, H., Ramachandran, S. and Manikantan, K. (2012). Circular sector dct based feature extraction for enhanced face recognition using histogram based dynamic gamma intensity correction, Proceedings of the CUBE International Information Technology Conference, ACM, pp. 74-81.

Gao, W., Cao, B., Shan, S., Chen, X., Zhou, D., Zhang, $X$. and Zhao, D. (2008). The cas-peal large-scale chinese face database and baseline evaluations, IEEE Transactions on Systems, Man, and CyberneticsPart A: Systems and Humans 38(1): 149-161.
Georghiades, A. S., Belhumeur, P. N. and Kriegman, D. J. (2001). From few to many: Illumination cone models for face recognition under variable lighting and pose, IEEE transactions on pattern analysis and machine intelligence 23(6): 643-660.

Gourier, N., Hall, D. and Crowley, J. L. (2004). Estimating face orientation from robust detection of salient facial structures, FG Net Workshop on Visual Observation of Deictic Gestures, Vol. 6.

Gross, R., Matthews, I., Cohn, J., Kanade, T. and Baker, S. (2010). Multi-pie, Image and Vision Computing 28(5): 807-813.

Gui, J., Sun, Z., Ji, S., Tao, D. and Tan, T. (2017). Feature selection based on structured sparsity: A comprehensive study, IEEE transactions on neural networks and learning systems 28(7): 1490-1507.

Hasan, M., Abdullah, S. N. H. S. and Othman, Z. A. (2013). Face recognition based on opposition particle swarm optimization and support vector machine, Signal and Image Processing Applications (ICSIPA), 2013 IEEE International Conference on, IEEE, pp. 417-424.

Hegade, P. P., Nishanth, R., Manikantan, K. and Ramachandran, S. (2013). Dwt-based face recognition using morphological edge detection as a pre-processing technique, Engineering (NUiCONE), 2013 Nirma University International Conference on, IEEE, pp. 1-6.

Holland, J. H. (1973). Genetic algorithms and the optimal allocation of trials, SIAM Journal on Computing 2(2): 88-105.

Huang, G. B., Ramesh, M., Berg, T. and LearnedMiller, E. (2007). Labeled faces in the wild: A database for studying face recognition in unconstrained environments, Technical report, Technical Report 07-49, University of Massachusetts, Amherst.

Islam, S., Bennamoun, M., Owens, R. A. and Davies, R. (2012). A review of recent advances in 3d earand expression-invariant face biometrics, ACM Computing Surveys (CSUR) 44(3): 14.

Jain, V. (2002). The indian face database/vidit jain, amitabha mukherjee, Kanpur: Indian Institute of Technology Kanpur .

Jesorsky, O., Kirchberg, K. J. and Frischholz, R. W. (2001). Robust face detection using the hausdorff distance, International Conference on Audio-and Video-Based Biometric Person Authentication, Springer, pp. 90-95.

Kallianpur, A. K., Kalyani, A., Naligay, S. E. and Kallimani, J. S. (2016). Facial recognition using discrete abc, Advances in Computing, Communications and Informatics (ICACCI), 2016 International Conference on, IEEE, pp. 2425-2429.

Kanan, H. R., Faez, K. and Hosseinzadeh, M. (2007). Face recognition system using ant colony optimization-based selected features, Computational Intelligence in Security and Defense Applications, 2007. CISDA 2007. IEEE Symposium on, IEEE, pp. 57-62. 
Karaboga, D. (2005). An idea based on honey bee swarm for numerical optimization, Technical report, Technical report-tro6, Erciyes university, engineering faculty, computer engineering department.

Kasinski, A., Florek, A. and Schmidt, A. (2008). The put face database, Image Processing and Communications 13(3-4): 59-64.

Kaur, H., Panchal, V. and Kumar, R. (2013a). A novel approach based on nature inspired intelligence for face feature extraction and recognition, Contemporary Computing (IC3), 2013 Sixth International Conference on, IEEE, pp. 149-153.

Kaur, H., Panchal, V. and Kumar, R. (2013b). A novel approach based on nature inspired intelligence for face feature extraction and recognition, Contemporary Computing (IC3), 2013 Sixth International Conference on, IEEE, pp. 149-153.

Keele, S. (2007). Guidelines for performing systematic literature reviews in software engineering, Technical report, Ver. 2.3 EBSE Technical Report. EBSE, sn.

Kennedy, J. and Eberhart, R. C. (1997). A discrete binary version of the particle swarm algorithm, Systems, Man, and Cybernetics, 1997. Computational Cybernetics and Simulation., 1997 IEEE International Conference on, Vol. 5, IEEE, pp. 4104-4108.

Khadhraoui, T., Ktata, S., Benzarti, F. and Amiri, H. (2016). Features selection based on modified pso algorithm for $2 \mathrm{~d}$ face recognition, Computer Graphics, Imaging and Visualization (CGiV), 2016 13th International Conference on, IEEE, pp. 99-104.

Khan, N. and Gupta, M. (2016). Face recognition system using improved artificial bee colony algorithm, Electrical, Electronics, and Optimization Techniques (ICEEOT), International Conference on, IEEE, pp. 3731-3735.

Khan, S. A., Usman, M. and Riaz, N. (2015). Face recognition via optimized features fusion, Journal of Intelligent \& Fuzzy Systems 28(4): 1819-1828.

Khashu, S., Vijayanagar, S., Manikantan, K. and Ramachandran, S. (2014). Face recognition using dual wavelet transform and filter-transformed flipping, Electronics and Communication Systems (ICECS), 2014 International Conference on, IEEE, pp. 17 .

Kim, S.-H., Oh, S.-K. and Kim, J.-Y. (2016). Design of face recognition system realized with the aid of pca-based rbfnn, Soft Computing and Intelligent Systems (SCIS) and 17th International Symposium on Advanced Intelligent Systems, 2016 Joint 8th International Conference on, IEEE, pp. 754-758.

Kishore, B., Rana, V. L., Manikantan, K. and Ramachandran, S. (2014). Face recognition using gabor-feature-based dft shifting, Industrial and Information Systems (ICIIS), 2014 9th International Conference on, IEEE, pp. 1-8.

Kitchenham, B. (2004). Procedures for performing systematic reviews, Keele, UK, Keele University 33(2004): 1-26.
Kodandaram, R., Mallikarjun, S., Krishnamuthan, M. and Sivan, R. (2015). Face recognition using truncated transform domain feature extraction., International Arab Journal of Information Technology (IAJIT) 12(3).

Kong, S. G., Heo, J., Abidi, B. R., Paik, J. and Abidi, M. A. (2005). Recent advances in visual and infrared face recognition-a review, Computer Vision and Image Understanding 97(1): 103-135.

Koza, J. R. (1992). Genetic programming: on the programming of computers by means of natural selection, Vol. 1, MIT press.

Krisshna, N. A., Deepak, V. K., Manikantan, K. and Ramachandran, S. (2014). Face recognition using transform domain feature extraction and pso-based feature selection, Applied Soft Computing 22: 141-161.

Lei, F., Lu, Y., Huang, W., Yu, L. and Jia, L. (2012). Fast static particle swarm optimization based feature selection for face detection, Computational Intelligence and Security (CIS), 2012 Eighth International Conference on, IEEE, pp. 401405 .

Liu, N. and Wang, H. (2006). Feature extraction with genetic algorithms based nonlinear principal component analysis for face recognition, Pattern Recognition, 2006. ICPR 2006. 18th International Conference on, Vol. 3, IEEE, pp. 461-464.

Loderer, M. and Pavlovičová, J. (2014). Optimization of LBP parameters, ELMAR (ELMAR), 2014 56th International Symposium, IEEE, pp. 1-4.

Lyons, M., Akamatsu, S., Kamachi, M. and Gyoba, J. (1998). Coding facial expressions with gabor wavelets, Automatic Face and Gesture Recognition, 1998. Proceedings. Third IEEE International Conference on, IEEE, pp. 200-205.

Mallipeddi, R. and Lee, M. (2012). Ensemble based face recognition using discriminant PCA features, Evolutionary Computation (CEC), 2012 IEEE Congress on, IEEE, pp. 1-7.

Martinez, A. M. (1998). The ar face database, CVC technical report .

McCulloch, W. S. and Pitts, W. (1943). A logical calculus of the ideas immanent in nervous activity, Bulletin of Mathematical Biophysics 5: 115-133.

Mollaee, M. and Moattar, M. H. (2016). Face recognition based on modified discriminant independent component analysis, Computer and Knowledge Engineering (ICCKE), 2016 6th International Conference on, IEEE, pp. 60-65.

Murthy, N. N., Raghunandana, R., Manikantan, K. and Ramachandran, S. (2012). Face recognition using dwt thresholding based feature extraction with laplacian-gradient masking as a preprocessing technique, Proceedings of the CUBE International Information Technology Conference, ACM, pp. 82-89. 
Naik, M. K. and Panda, R. (2016). A novel adaptive cuckoo search algorithm for intrinsic discriminant analysis based face recognition, Applied Soft Computing 38: 661-675.

Navau, P. O. A., da Silva, A. S. and de Azevedo, R. J. (2013). Documento de Área 2013, Master's thesis.

Nebti, S. and Boukerram, A. (2017). Swarm intelligence inspired classifiers for facial recognition, Swarm and Evolutionary Computation 32: $150-166$.

Nema, S. and Thakur, S. (2015). Improved particle swarm optimization approach for classification by using lda, Intelligent Systems and Control (ISCO), 2015 IEEE 9th International Conference on, IEEE, pp. 1-5.

Nischal, K., Nayak, M. P., Manikantan, K. and Ramachandran, S. (2013). Face recognition using entropy-augmented face isolation and image folding as pre-processing techniques, India Conference (INDICON), 2013 Annual IEEE, IEEE, pp. 16.

Niveditha, G., Sharmila, B., Manikantan, K. and Ramachandran, S. (2015). Dwt-based face recognition using fast walsh hadamard transform and chiral image superimposition as pre-processing techniques, Electronics and Communication Systems (ICECS), 2015 2nd International Conference on, IEEE, pp. 7-14.

Ochoa-Villegas, M. A., Nolazco-Flores, J. A., BarronCano, O. and Kakadiaris, I. A. (2015). Addressing the illumination challenge in two-dimensional face recognition: a survey, IET Computer Vision 9(6): 978992.

Oh, S.-K., Yoo, S.-H. and Pedrycz, W. (2013). Design of face recognition algorithm using pcalda combined for hybrid data pre-processing and polynomial-based rbf neural networks: Design and its application, Expert Systems with Applications 40(5): 1451-1466.

Oh, S.-K., Yoo, S.-H. and Pedrycz, W. (2016). A comparative study of feature extraction methods and their application to p-rbf nns in face recognition problem, Fuzzy Sets and Systems 305: $131-148$.

Pan, H., Zhu, Y. and Xia, L. (2013). Fusing multifeature representation and pso-adaboost based feature selection for reliable frontal face detection, Image Processing (ICIP), 2013 2oth IEEE International Conference on, IEEE, pp. 2998-3002.

Panda, R. and Naik, M. K. (2015). A novel adaptive crossover bacterial foraging optimization algorithm for linear discriminant analysis based face recognition, Applied Soft Computing 30: 722736.

Parpinelli, R. S. and Lopes, H. S. (2011). New inspirations in swarm intelligence: a survey, International Journal of Bio-Inspired Computation 3(1): 1-16.

Passino, K. M. (2002). Biomimicry of bacterial foraging for distributed optimization and control, IEEE control systems 22(3): 52-67.
Petersen, K., Feldt, R., Mujtaba, S. and Mattsson, M. (2008). Systematic mapping studies in software engineering., EASE, Vol. 8, pp. 68-77.

Pham, D., Ghanbarzadeh, A., Koc, E., Otri, S., Rahim, S. and Zaidi, M. (2011). The bees algorithm-a novel tool for complex optimisation, Intelligent Production Machines and Systems-2nd I* PROMS Virtual International Conference (3-14 July 2006), sn.

Phillips, P. J., Moon, H., Rizvi, S. A. and Rauss, P. J. (2000). The feret evaluation methodology for facerecognition algorithms, IEEE Transactions on pattern analysis and machine intelligence 22(10): 1090-1104.

Plichoski, G. F., Parpinelli, R. S. and Chidambaram, C. (2017). Optimizing a homomorphic filter for illumination compensation in face recognition using population-based algorithms, XIII Workshop de Visão Computacional .

Prabhu, N. S., Kesari, T. N., Manikantan, K. and Ramachandran, S. (2013). Face recognition using eccentricity-range based background removal and multi-scaled fusion as pre-processing techniques, Engineering (NUiCONE), 2013 Nirma University International Conference on, IEEE, pp. 1-6.

Rao, N. M., Aditya, S., Manikantan, K. and Ramachandran, S. (2014). Face recognition using entropy based feature enhancement and parallel dual pose testing, Medical Imaging, $m$-Health and Emerging Communication Systems (MedCom), 2014 International Conference on, IEEE, pp. 352-357.

Rao, S. and Rao, M. B. (2016). A novel triangular dct feature extraction for enhanced face recognition, Intelligent Systems and Control (ISCO), 2016 1oth International Conference on, IEEE, pp. 1-6.

Rashedi, E., Nezamabadi-pour, H. and Saryazdi, S. (2009). Gsa: A gravitational search algorithm, Information Sciences 179(13): 2232 - 2248. Special Section on High Order Fuzzy Sets.

Rinky, B., Mondal, P., Manikantan, K. and Ramachandran, S. (2012). Dwt based feature extraction using edge tracked scale normalization for enhanced face recognition, Procedia Technology 6: $344-353$.

Roohi, S. T., Sachin, D. S., Manikantan, K. and Ramachandran, S. (2013). Feature accentuation using uniform morphological correction as pre-processing technique for dwt based face recognition, Procedia Technology 10: 690-698.

Sah, R., Shreeja, B., Manikantan, K. and Ramachandran, S. (2015). Entropic-gwt based feature extraction and lbpso based feature selection for enhanced face recognition, Communications and Signal Processing (ICCSP), 2015 International Conference on, IEEE, pp. 0180-0184.

Samaria, F. S. and Harter, A. C. (1994). Parameterisation of a stochastic model for human face identification, Applications of Computer Vision, 1994., Proceedings of the Second IEEE Workshop on, IEEE, pp. 138-142. 
Sattiraju, M., Manikandan, M. V., Manikantan, K. and Ramachandran, S. (2013). Adaptive bpso based feature selection and skin detection based background removal for enhanced face recognition, Computer Vision, Pattern Recognition, Image Processing and Graphics (NCVPRIPG), 2013 Fourth National Conference on, IEEE, pp. 1-4.

Scheenstra, A., Ruifrok, A. and Veltkamp, R. (2005). A survey of $3 \mathrm{~d}$ face recognition methods, Audioand Video-Based Biometric Person Authentication, Springer, pp. 325-345.

Shanbhag, S. S., Bargi, S., Manikantan, K. and Ramachandran, S. (2014). Face recognition using wavelet transforms-based feature extraction and spatial differentiation-based pre-processing, Science Engineering and Management Research (ICSEMR), 2014 International Conference on, IEEE, pp. 1-8.

Shen, X., Yi, Y., Dong, W., Guo, S., Li, J. and Chen, F. (2015). Face detection based on particle swarm optimisation-free entropy minimisation, International Journal of Computing Science and Mathematics 6(2): 129-138.

Shetty, S., Kelkar, P., Manikantan, K. and Ramachandran, S. (2013). Shift invariance based feature extraction and weighted bpso based feature selection for enhanced face recognition, Procedia Technology 10: 822-830.

Shieh, M.-Y., Chiou, J.-S., Hu, Y.-C. and Wang, K.Y. (2014). Applications of pca and svm-pso based real-time face recognition system, Mathematical Problems in Engineering 2014.

Soumya, S., Manikantan, K., Ramachandran, S. et al. (2013). Face recognition using wavelet filtering and dual pose testing, India Conference (INDICON), 2013 Annual IEEE, IEEE, pp. 1-6.

Spacek, L. (2002). University of essex face database.

Storn, R. and Price, K. (1997). Differential evolution-a simple and efficient heuristic for global optimization over continuous spaces, Journal of global optimization 11(4): 341-359.

Surabhi, A., Parekh, S. T., Manikantan, K. and Ramachandran, S. (2012). Background removal using $\mathrm{k}$-means clustering as a preprocessing technique for dwt based face recognition, Communication, Information \& Computing Technology (ICCICT), 2012 International Conference on, IEEE, pp. 1-6.

Thomaz, C. E. and Giraldi, G. A. (2010). A new ranking method for principal components analysis and its application to face image analysis, Image and Vision Computing 28(6): 902-913.

Trinh, T. D., Kim, J. Y. and Na, S. Y. (2014). Enhanced face recognition by fusion of global and local features under varying illumination, IT Convergence and Security (ICITCS), 2014 International Conference on, IEEE, pp. 1-4.

Valuvanathorn, S., Nitsuwat, S. and Huang, M. L. (2012). Multi-feature face recognition based on psosvm, ICT and Knowledge Engineering (ICT \& Knowledge
Engineering), 2012 1oth International Conference on, IEEE, pp. 140-145.

Varadarajan, K., Suhasini, P., Manikantan, K. and Ramachandran, S. (2015). Face recognition using block based feature extraction with czt and goertzel-algorithm as a preprocessing technique, Procedia Computer Science 46: 1458-1467.

Varun, R., Kini, Y. V., Manikantan, K. and Ramachandran, S. (2015). Face recognition using hough transform based feature extraction, Procedia Computer Science 46: 1491-1500.

Venkatesan, S. and Madane, S. S. R. (2010). Face recognition system with genetic algorithm and ant colony optimization, International Journal of Innovation, Management and Technology 1(5): 469.

Vidya, V., Farheen, N., Manikantan, K. and Ramachandran, S. (2012). Face recognition using threshold based dwt feature extraction and selective illumination enhancement technique, Procedia Technology 6: 334-343.

Vignolo, L. D., Milone, D. H. and Scharcanski, J. (2013). Feature selection for face recognition based on multi-objective evolutionary wrappers, Expert Systems with Applications 40(13): 5077-5084.

Vignolo, L., Milone, D., Behaine, C. and Scharcanski, J. (2012). An evolutionary wrapper for feature selection in face recognition applications, Systems, Man, and Cybernetics (SMC), 2012 IEEE International Conference on, IEEE, pp. 1286-1290.

Vora, A., Raj, A., Manikantan, K. and Ramachandran, S. (2014). Enhanced face recognition using 8-connectivity-of-skin-region and standarddeviation-based-pose-detection as preprocessing techniques, Medical Imaging, $m$-Health and Emerging Communication Systems (MedCom), 2014 International Conference on, IEEE, pp. 364-369.

Wechsler, H., Phillips, J. P., Bruce, V., Soulie, F. F. and Huang, T. S. (2012). Face recognition: From theory to applications, Vol. 163, Springer Science \& Business Media.

Weyrauch, B., Heisele, B., Huang, J. and Blanz, V. (2004). Component-based face recognition with 3d morphable models, Computer Vision and Pattern Recognition Workshop, 2004. CVPRW'04. Conference on, IEEE, pp. 85-85.

Xiao, T., Ren, D., Lei, S., Zhang, J. and Liu, X. (2014). Based on grid-search and pso parameter optimization for support vector machine, Intelligent Control and Automation (WCICA), 2014 11th World Congress on, IEEE, pp. 1529-1533.

Yadav, D., Vatsa, M., Singh, R. and Tistarelli, M. (2013). Bacteria foraging fusion for face recognition across age progression, Proceedings of the IEEE Conference on Computer Vision and Pattern Recognition Workshops, pp. 173-179.

Yaji, G. S., Sarkar, S., Manikantan, K. and Ramachandran, S. (2012). Dwt feature extraction based face recognition using intensity mapped unsharp masking and laplacian of gaussian filtering with scalar multiplier, Procedia Technology 6: $475-484$. 
Yang, X.-S. and Deb, S. (2009). Cuckoo search via lévy flights, Nature \& Biologically Inspired Computing, 2009. NaBIC 2009. World Congress on, IEEE, pp. 210214.

YIN, H., FU, P. and SUN, Z. (2014). Face feature selection and recognition using separability criterion and binary particle swarm optimization algorithm, Chinese Journal of Electronics 2: 025.

Yin, H., Qiao, J., Fu, P. and Xia, X. (2014). Face feature selection with binary particle swarm optimization and support vector machine, J. Inf. Hiding Multimed. Signal Process 5(4): 731-739.

Yoo, S.-H., Oh, S.-K. and Pedrycz, W. (2013). Design of face recognition algorithm realized with feature extraction from 2d-lda and optimized polynomialbased rbf nns, IFSA World Congress and NAFIPS Annual Meeting (IFSA/NAFIPS), 2013 Joint, IEEE, pp. 655660.

Zhang, Y. and Peng, H. (2016). One sample per person face recognition based on particle swarm optimisation, IET Signal Processing 10(2): 100-105.

Zhang, Y., Wang, S. and Ji, G. (2015). A comprehensive survey on particle swarm optimization algorithm and its applications, Mathematical Problems in Engineering 2015.

Zhao, W., Chellappa, R., Phillips, P. J. and Rosenfeld, A. (2003). Face recognition: A literature survey, ACM computing surveys (CSUR) 35(4): 399-458.

Zheng, W.-S., Lai, J.-H. and Yuen, P. C. (2005). Ga-fisher: a new lda-based face recognition algorithm with selection of principal components, IEEE Transactions on Systems, Man, and Cybernetics, Part B (Cybernetics) 35(5): 1065-1078.

Zou, D. and Zhang, H. (2016). Face recognition method based on 2dlda and svm optimated by pso algorithm, Advances in Intelligent Systems Research 130. 